\title{
Evolutionary patterns of carbohydrate transport and metabolism in Halomonas boliviensis as derived from its genome sequence: influences on polyester production
}

Daniel Guzmán 1,2, Andrea Balderrama-Subieta1', Carla Cardona-Ortuño', Mónica Guevara-Martínez', Nataly Callisaya-Quispe ${ }^{1}$ and Jorge Quillaguamán ${ }^{1 *}$

\begin{abstract}
Background: Halomonas boliviensis is a halophilic bacterium that is included in the $\gamma$-Proteobacteria sub-group, and is able to assimilate different types of carbohydrates. H. boliviensis is also able to produce poly(3hydroxybutyrate) (PHB) in high yields using glucose as the carbon precursor. Accumulation of PHB by microorganisms is induced by excess of intracellular NADH.

The genome sequences and organization in microorganisms should be the result of evolution and adaptation influenced by mutation, gene duplication, horizontal gen transfer (HGT) and recombination. Furthermore, the nearly neutral theory of evolution sustains that genetic modification of DNA could be neutral or selected, albeit most mutations should be at the border between neutrality and selection, i.e. slightly deleterious base substitutions in DNA are followed by a slightly advantageous substitutions.
\end{abstract}

Results: This article reports the genome sequence of $\mathrm{H}$. boliviensis. The chromosome size of $\mathrm{H}$. boliviensis was 4 119979 bp, and contained 3863 genes. A total of 160 genes of $\mathrm{H}$. boliviensis were related to carbohydrate transport and metabolism, and were organized as: 70 genes for metabolism of carbohydrates; 47 genes for ABC transport systems and 43 genes for TRAP-type C4-dicarboxylate transport systems. Protein sequences of $H$. boliviensis related to carbohydrate transport and metabolism were selected from clusters of orthologous proteins (COGs). Similar proteins derived from the genome sequences of other 41 archaea and 59 bacteria were used as reference. We found that most of the 160 genes in H. boliviensis, c.a. 44\%, were obtained from other bacteria by horizontal gene transfer, while 13\% of the genes were acquired from haloarchaea and thermophilic archaea, only $34 \%$ of the genes evolved among Proteobacteria and the remaining genes encoded proteins that did not cluster with any of the proteins obtained from the reference strains. Furthermore, the diversity of the enzymes derived from these genes led to polymorphism in glycolysis and gluconeogenesis. We found further that an optimum ratio of glucose and sucrose in the culture medium of $\mathrm{H}$. boliviensis favored cell growth and PHB production.

Conclusions: Results obtained in this article depict that most genetic modifications and enzyme polymorphism in the genome of $\mathrm{H}$. boliviensis were mainly influenced by HGT rather than nearly neutral mutations. Molecular adaptation and evolution experienced by $\mathrm{H}$. boliviensis were also a response to environmental conditions such as the type and amount of carbohydrates in its ecological niche. Consequently, the genome evolution of $\mathrm{H}$. boliviensis showed to be strongly influenced by the type of microorganisms, genetic interaction among microbial species and its environment. Such trend should also be experienced by other prokaryotes. A system for PHB production by H. boliviensis that takes into account the evolutionary adaptation of this bacterium to the assimilation of

\footnotetext{
* Correspondence: jorgeqs@supernet.com.bo

${ }^{1}$ Centro de Biotecnología, Facultad de Ciencias y Tecnología, Universidad

Mayor de San Simón, Cochabamba, Bolivia

Full list of author information is available at the end of the article
} 
combinations of carbohydrates suggests the feasibility of a bioprocess economically viable and environmentally friendly.

Keywords: Halomonas boliviensis, Halophilic bacterium, Halomonas, Halomonadaceae, Biopolyesters, Polyhydroxyalkanoates, Genome evolution, Population genetics

\section{Background}

Cellular evolution and adaptation have imprinted patterns in microbial genomes through mutation, gene duplication, horizontal gen transfer (HGT) and recombination $[1,2]$. The genomes of microorganisms of the three domains of life have experienced such genetic modifications to succeed on their permanence in a particular habitat, where environmental conditions and the size of the microbial populations might influence the organization and number of genes in a particular species throughout the time $[1,3]$. Furthermore, the nearly neutral theory of evolution points out that genetic modification of DNA could be neutral or selected, albeit most mutations should be at the border between neutrality and selection, i.e. slightly deleterious base substitutions in DNA are followed by a slightly advantageous substitutions [1].

The increasing number of genome sequences of different organisms is helping to discern how microbial species diverged. Recent reports on the evolutionary traits followed by different bacteria and archaea have demonstrated that the transfer of genes among these organisms, also referred as horizontal gene transfer, has led to net-like relationships among their genomes $[2,4,5]$. Nevertheless, the phylogenetic association among prokaryotes derived from the sequences of proteins encoded by 102 different genes was consistent to the taxonomic differentiation observed when 16 rRNA sequences of microorganisms are analyzed [4]. The 102 proteins were mainly related to translation and transcription, although proteins involved in the transport and metabolism of amino acids, metal ions and carbohydrates revealed such taxonomic information as well [4].

The aforementioned studies included the genome sequences of extremely halophilic archaea such as Haloarcula marismortui, Haloquadratum walsbyi and a Halobacterium sp. [4]. These studies on the genome sequences did not include halophilic bacteria. However, a report on the genes of poly(3-hydroxybutyrate) (PHB) polymerases, $\mathrm{PHB}$ depolymerases and ectoine synthesis by Halomonas sp. TD01, a halophilic bacterium, suggested that HGT has a role to play on the genome organization of the microorganism [6]. Halophilic microorganisms require salt $(\mathrm{NaCl})$ to grow; a halophile should grow optimally at $\mathrm{NaCl}$ concentrations of $5 \%(\mathrm{w} / \mathrm{v})$ or higher, and tolerate at least $10 \%(\mathrm{w} / \mathrm{v})$ salt [7]. There are five genome sequences of halophilic bacteria available in public data bases. The sequences of Chromohalobacter salexigens and
Halorhodospira halophila were first published followed by the sequence of Halomonas elongata [8,9], Halomonas sp. TD01 [6] and Halomonas sp. HAL1 [10]. Chromohalobacter and Halomonas species are included in the family Halomonadaceae within the $\gamma$-Proteobacteria subgroup. The family Halomonadaceae contains only halophilic and halotolerant aerobic heterotrophs; some of them are able

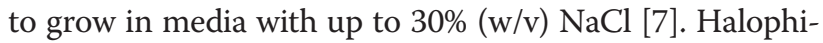
lic bacteria maintain low concentrations of salt intracellularly by accumulating organic compounds of low molecular weight, also known as osmolytes or "compatible solutes" such as ectoine [11].

Understanding the evolution and levels of polymorphism among genes is attracting much attention in evolutionary biology and biotechnology. Evolution of energy-producing pathways, particularly glycolysis and gluconeogenesis, posses relevance since they determine the type of carbon sources that a species is able to assimilate, and link to metabolic routs that may generate compounds of biotechnological interest [12]. Theories on the evolution of the metabolisms of organisms consider that enzyme polymorphism-alleles for the different enzymes or allozymesin metabolic pathways was related to genetic mutations [12-14]. A proposal states that the fitness of the pathways associated with an increasing flux is influenced by selected mutations of genes that enhance enzyme activities, albeit enzyme improvements do not continue indefinitely [12,14]. Mutations will reach a point at which the incremental gains of fitness for a new mutation will be equaled by the noise caused by the random genetic variation [12,14]. At this stage, the genes or enzymes might evolve under a nearly neutral trend $[12,14]$. Moreover, metabolic control in the organisms is also to regulate molecular evolution as well $[12,14]$. The proposal assumes no contextual changes such as a change in the functional conditions of an enzyme originated by either epistasis or the environment; or a change in the effective population size of the species [12].

Halomonas boliviensis is a halophilic bacterium that can develop under a wide range of $\mathrm{NaCl}$ concentrations (i.e. $0-25 \%(\mathrm{w} / \mathrm{v})), \mathrm{pH}(5-11)$ and temperatures $\left(0-45^{\circ} \mathrm{C}\right)$ [15]. It can also assimilate several carbohydrates as carbon source for growth [15]. Bioprocesses have been designed to attain high productivities of a polyester and osmolytes by $H$. boliviensis using glucose as the carbon precursor $[16,17]$. The polyester accumulated by the bacterium is poly(3-hydroxybutyrate) (PHB), which is used as carbon and energy reservoir [18]. PHB is synthesized 
by several bacteria from acetyl-CoA when an excess of NADH is present in the bacterial cytoplasm [19]. Such excess can be generated when a high concentration of a carbon source is added to a culture medium and cell growth is limited by the depletion of an essential nutrient, e.g. nitrogen, oxygen, trace elements among others [19]. PHB is attracting much attention in biotechnology because it is a biodegradable plastic-like material, and possesses potential in biomedical applications such as tissue engineering, organ transplants and drug delivery systems [20]. Moreover, the efficiency and economics of the manufacturing process of PHB are determined by the carbon source, fermentation process, and downstream processing of the polymer. The development of cultivation conditions for microorganisms that allow high $\mathrm{PHB}$ content and productivity from cheap and renewable carbon sources is therefore important [21,22].

The present research work reports the genome sequence of $H$. boliviensis. It also depicts the evolutionary trends that proteins of $H$. boliviensis have experienced to allow the transport of carbohydrates and their assimilation to achieve acetyl-CoA. The conclusions drawn from these studies were used to create an alternative production system of PHB by $H$. boliviensis using a combination of carbohydrates. This system should lead to a more economically and environmentally beneficial bioprocess.

\section{Methods}

\section{Genome sequencing}

The fine high coverage genome sequence, gene prediction, repetitive sequence, COGs and KEGG annotation of Halomonas boliviensis $\mathrm{LC}^{\mathrm{T}}\left(=\mathrm{DSM} 15516^{\mathrm{T}}\right)$ were obtained at BGI-Hongkong Co., Hong Kong. For this, Illumina HiSeq 2000 technology was used to conduct paired-end sequencing for DNA samples, and constructed a 1,000 bp library with extended data of $500 \mathrm{Mb}$. Genome coverage based on k-mer was $95.4 \%$, and genome coverage based on reads mapping was $99.9 \%$. Glimmer 3.0 software package was used to conduct de novo gene prediction [23]. The functional annotation was accomplished by analysis of protein sequences. Genes of $H$. boliviensis were aligned to others in databases to attain its corresponding functional annotation. To ensure the biological meaning, only one high-quality information as annotation to the genes from many results was chosen. BLAST was used to accomplish functional annotation combined with different databases. BLAST version: blastall 2.2.21 software (provided by the National Center for Biotechnology Information, NCBI) was used for these studies. Alignment results were obtained using the following databases: KEGG, COG, SwissProt, TrEMBL, NR. This whole genome shotgun project was deposited at DDBJ, EMBL and GenBank under the accession number
AGQZ00000000. The version described in this paper is the first version, AGQZ01000000.

\section{Evolutionary analysis}

A total of 6,901 alignments of clusters of orthologous proteins (COGs) of 59 bacteria and 41 archaea, as classified in COGs [24] and EggNOG [25] data bases, were gently provided by Puigbò, Wolf and Koonin (2009). The protein sequences of these 100 microorganisms were used as reference for the evolutionary analysis. Protein sequences of $H$. boliviensis related to carbohydrate transport and metabolism were selected and aligned along with the references for each corresponding COG (Additional file 1: Table S1, supplementary data) using the Muscle program [26] included in the MEGA 5 software package [27] with default parameters. Unrooted maximum likelihood phylogenetic trees were constructed using MEGA 5 under a WAG with frequencies $(+\mathrm{F})$ model, with uniform mutation rates among amino acid sites and complete deletion of gaps and missing data.

\section{Analysis and assembly of supernetworks}

Supernetworks were constructed by combining the phylogenetic trees of proteins of the glycolysis and gluconeogenesis metabolisms in $H$. boliviensis and reference strains using the SplitsTree4 program $[28,29]$ with default parameters. Three analyses were performed for these studies: 1) A supernetwork obtained from three COGs $(0126,0149$ and 0837). Both COG0126 and COG0149 are considered among the 102 genes that contain taxonomic information that discriminate well bacteria and archaea in already known families and genera [4]; 2) A supernetwork obtained after combining six COGs (0126, 0149, 0837, 0469, 0696 and 837); and 3) A supernetwork obtained after combining twenty two COGs $(0057,0126,0148$, 0149, 0166, 0191, 0205, 0235, 0365, 0469, 0508, 0696, 0837, 1012, 1063, 1109, 1249, 1454, 1866, 2017, 2609 and 4993). Supernetworks were analyzed according to method described by Huson et al. in 2006 [28].

\section{Culture media composition}

Seed culture and PHB production media were formulated as described previously [16]. Seed culture contained\% (w/v): $\mathrm{NaCl}, 2.5 ; \mathrm{MgSO}_{4} \cdot 7 \mathrm{H}_{2} \mathrm{O}, 0.25 ; \mathrm{K}_{2} \mathrm{HPO}_{4}, 0.05$; $\mathrm{NH}_{4} \mathrm{Cl}, 0.23 ; \mathrm{FeSO}_{4} \bullet 7 \mathrm{H}_{2} \mathrm{O}, 0.005$; sucrose 1 ; monosodium glutamate (MSG), 0.3 and TRIS, 1.5. The PHB production medium included \% (w/v): $\mathrm{NaCl}, 2.5 ; \mathrm{MgSO}_{4} \cdot 7 \mathrm{H}_{2} \mathrm{O}, 0.5$; $\mathrm{K}_{2} \mathrm{HPO}_{4}, 0.22 ; \mathrm{NH}_{4} \mathrm{Cl}, 0.4 ; \mathrm{FeSO}_{4} \cdot 7 \mathrm{H}_{2} \mathrm{O}, 0.005 ; \mathrm{MSG}, 0.2$; and the following concentration of carbohydrates $\%(\mathrm{w} / \mathrm{v})$ : 1) 2.5 sucrose, 2) 2.0 sucrose and 0.5 glucose, 3) 1.5 sucrose and 1 glucose, 4) 1.0 sucrose and 1.5 glucose, 5) 0.3 sucrose, 0.7 glucose and 1.5 dried molasses and 6) 2.5 dried molasses for 6 different assays, respectively. The 
composition of the molasses used was $78.1 \%$ sucrose, $15.3 \%$ glucose and $6.6 \%$ of other uncharacterized solids. A low amount of MSG is added to the production medium to induce its depletion by $H$. boliviensis during the cultivation.

\section{$H$. boliviensis growth and PHB production in flasks}

$H$. boliviensis was grown in $100 \mathrm{ml}$ of seed culture medium in 1,000-ml flasks with rotary shaking at $220 \mathrm{rpm}$, $30^{\circ} \mathrm{C}$ for $13 \mathrm{~h}$. The $\mathrm{pH}$ of the medium was adjusted to 7.5 using concentrated $\mathrm{HCl}$. Subsequently, $5 \mathrm{ml}$ of the seed culture were inoculated in 1,000-ml Erlenmeyer flasks containing $95 \mathrm{ml}$ of $\mathrm{PHB}$ production medium. The $\mathrm{pH}$ of the $\mathrm{PHB}$ production medium was initially adjusted to 7.5 using $5 \mathrm{M} \mathrm{NaOH}$. The cultures were incubated at $35^{\circ} \mathrm{C}$ with shaking at $220 \mathrm{rpm}$, and samples were withdrawn at different time intervals during the cultivation.

\section{Quantitative analyses}

Cell dry weight (CDW) and PHB content in H. boliviensis were determined as reported previously [18]. Residual cell mass (RCM) concentration was calculated as the difference between the CDW and PHB concentration, while PHB content (wt\%) was obtained as the percentage of the ratio of $\mathrm{PHB}$ concentration to the $\mathrm{CDW}$ as defined by Lee et al. in 2000 [30]. All analyses were performed in triplicate.

Glutamate concentration was determined by high performance liquid chromatography (HPLC) analysis, as described previously [31], using a Perkin-Elmer HPLC system with an Aminex HPX-87 C column (Biorad) and a UV detector at $65^{\circ} \mathrm{C}$. Calcium chloride solution $(5 \mathrm{mM})$ was used as mobile phase at a flow rate of $0.5 \mathrm{ml} / \mathrm{min}$. Glutamate was monitored at $210 \mathrm{~nm}$. Glucose and sucrose were determined using the same HPLC system with a Polypore CA column (Perkin-Elmer), a RI detector at $80^{\circ} \mathrm{C}$ and water as mobile phase at a flow rate of $0.3 \mathrm{ml} / \mathrm{min}$.

\section{Results and discussion}

\section{Genome of $H$. boliviensis}

Table 1 provides a description of the genome composition of $H$. boliviensis. The chromosome size of $H$. boliviensis (4 $119979 \mathrm{bp)}$ ) was slightly longer than those determined for H. elongata (4 061296 bp) [9], Halomonas sp. TD01 (4 092837 bp) [6] and Chromohalobacter salexigens (3 696649 bp) (Accession number: CP000285.1). The \% of $\mathrm{G}+\mathrm{C}$ content showed in Table 1 is similar to that determined experimentally for $H$. boliviensis, i.e. 52.6\% [15], and is lower than that found for the genome of $H$. elongata $(63.6 \%)$ and that evaluated for the description of C. salexigens (64.2\%) [32]. Such wide difference between the $\mathrm{G}+\mathrm{C}$ content of different Halomonas and Chromohalobacter species is a feature of the family Halomonadaceae [33]. Moreover, the genes constitute most part of the
Table 1 Genome of H.boliviensis

\begin{tabular}{ll}
\hline Chromosome & 1 \\
DNA, total number of bases & 4119979 \\
$\%$ G+C content & 54.69 \\
Number of genes & 3863 \\
Length occupied by genes (bp) & 3673824 \\
$\%$ G+C content in the gene region & 55.64 \\
$\%$ Gene/Chromosome & 89.17 \\
Length occupied by the intergenic region (bp) & 446146 \\
$\%$ G+C content in the intergenic region & 46.86 \\
\% Intergenic length/Chromosome & 10.82 \\
\hline
\end{tabular}

chromosome of $H$. boliviensis and the $\% \mathrm{G}+\mathrm{C}$ content for the region containing the genes was similar to that found in its chromosome (Table 1). On the other hand, the number of genes in the genome of $H$. boliviensis $(3863)$ is slightly higher than that reported for H. elongata (3 555) [9].

Inferring the evolution of proteins involved in the uptake and metabolism of carbohydrates

Protein sequences of $H$. boliviensis related to carbohydrate transport and metabolism were obtained from clusters of orthologous proteins (COGs), as classified in COGs and EggNOG data bases [24,25]. A total of 160 genes of $H$. boliviensis encoded proteins for these clusters: 70 genes were related to the metabolism of carbohydrates; 47 genes were related to ABC transport systems and encoded 14 permease proteins, 23 ATPase proteins and 10 periplasmic proteins; and 43 genes were related to TRAP-type C4-dicarboxylate transport systems and encoded 15 large permease proteins, 11 small permease proteins and 17 periplasmic proteins (Additional file 1: Table S1, supplementary data). Similar proteins were selected from COGs derived from the genome sequences of other 41 archaea and 59 bacteria. To perform evolutionary analyses, unrooted phylogenetic trees were constructed based on a maximum likelihood approach using the sequences of the proteins of $H$. boliviensis and proteins of other 100 microorganisms for each corresponding COG.

Figure 1 presents three phylogenetic trees that were selected to exemplify the genetic modifications experienced by the genome of $H$. boliviensis. Figure 1 shows a phylogenetic tree for a COG related to the ABC type transport system for ribose, xylose, arabinose and galactoside. $H$. boliviensis has three alternative forms of genes, i.e. alleles, for this tree. The first allele $(H$. boliviensis A1) was clustered with thermophilic archaea (Figure 1), hence implying a long distance HGT [5]. After comparing the closest identities of this allele to other sequences in pubic data bases, we found that the sequence corresponded to a periplasmic binding protein. We also found that the 


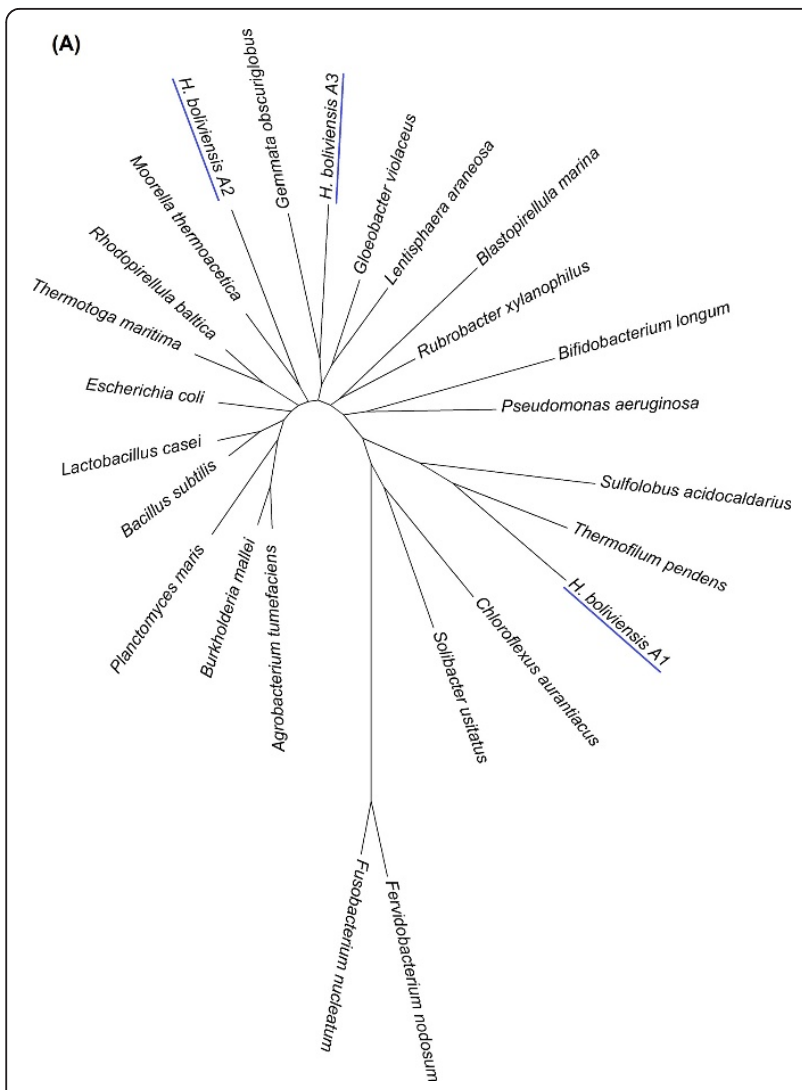

Figure 1 Phylogenetic tree relating clusters of orthologous protein sequences (COGs):ABC type transport system for ribose, xylose, arabinose and galactoside, COG1172. The tree was constructed under a maximum likelihood approach using MEGA 5 software and the WAG with frequencies (+F) model. GeneBank accession numbers of the sequences included in the figure are provided in Additional file 2: Table S2.

amount of acidic amino acids of $H$. boliviensis A1 (10.1\% of the total residues in the protein) was significantly higher than the basic amino acids (3.5\%), resulting in a ratio of 2.9 of acidic to basic amino acids. Various scientific articles reported that some extracellular and periplasmic enzymes of halophilic bacteria tolerate high temperatures, and possess a relatively high content of acidic amino acids $[8,34]$. The result obtained in Figure 1 denotes that $H$. boliviensis had originally obtained the binding protein from a thermophile and it diverged later. The second allele (H. boliviensis A2) was also identified as a periplasmic binding protein with similar functions to those of the first allele. However, the second allele shared a closer affiliation to proteins of bacteria other than Proteobacteria (Figure 1). The ratio of acidic $(9.4 \%$ of the total residues in the protein) to basic (5.5\%) amino acids for allele 2 was 1.7 . $H$. boliviensis is a microorganism that is able to grow at low $\left(0^{\circ} \mathrm{C}\right)$ and high $\left(45^{\circ} \mathrm{C}\right)$ temperatures, whereby it should be useful for this organism to hold two different proteins that can accomplish a similar task; one active at low temperatures and the second active at high temperatures. The third allele ( $H$. boliviensis A3) was clustered along with trans-membrane proteins of Planctomycetes, Cyanobateria and Lentisphaerae (Figure 1). Both allele 2 and 3 suggest HGT among bacteria [5].

On the other hand, Figure 2 depicts a phylogenetic tree for a COG corresponding to keto-3-deoxy-6-phosphogluconate aldolases. The first allele of $H$. boliviensis in this phylogenetic tree was closely related to proteins of halophilic archaea, while the second diverged among enzymes of Proteobacteria with a close relationship to $\gamma$-Proteobacteria (Figure 2). H. boliviensis has also adapted to a wide range of $\mathrm{NaCl}$ concentrations. At different $\mathrm{NaCl}$ concentrations, $H$. boliviensis should thrive in environments with distinct type of microorganisms varying from non-halophilic to extreme halophilic archaea [7]. H. boliviensis showed to be able to acquire genes from other organisms that share its habitat (Figures 1,2). Moreover, Figure 3 depicts two alleles that are closely related to proteins of $\gamma$-Proteobacteria, as might be expected. The same evolutionary analyses were followed with all COGs related to carbohydrate transport and metabolism. The results obtained are summarized in Additional file 1: Table S1 and in Figure 4. Interestingly, most of the proteins (44\%) of $\mathrm{H}$. boliviensis involved in carbohydrate transport and metabolism were

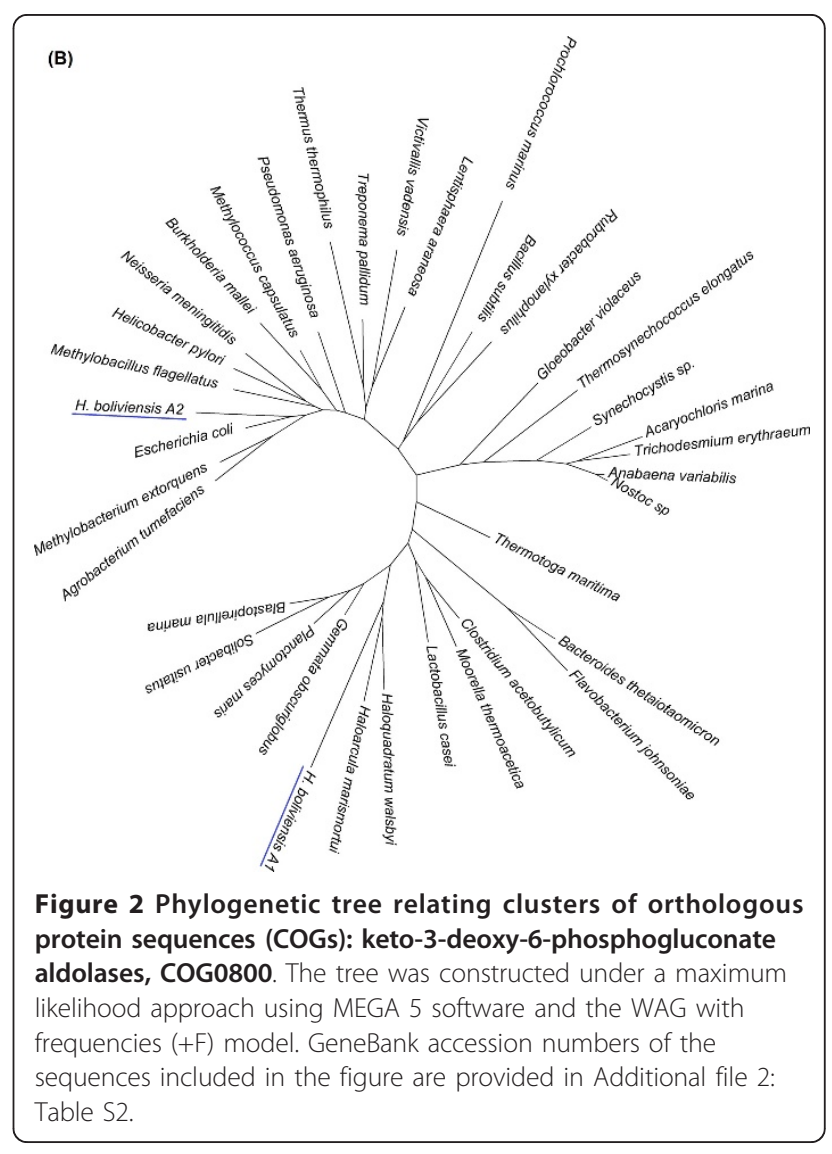




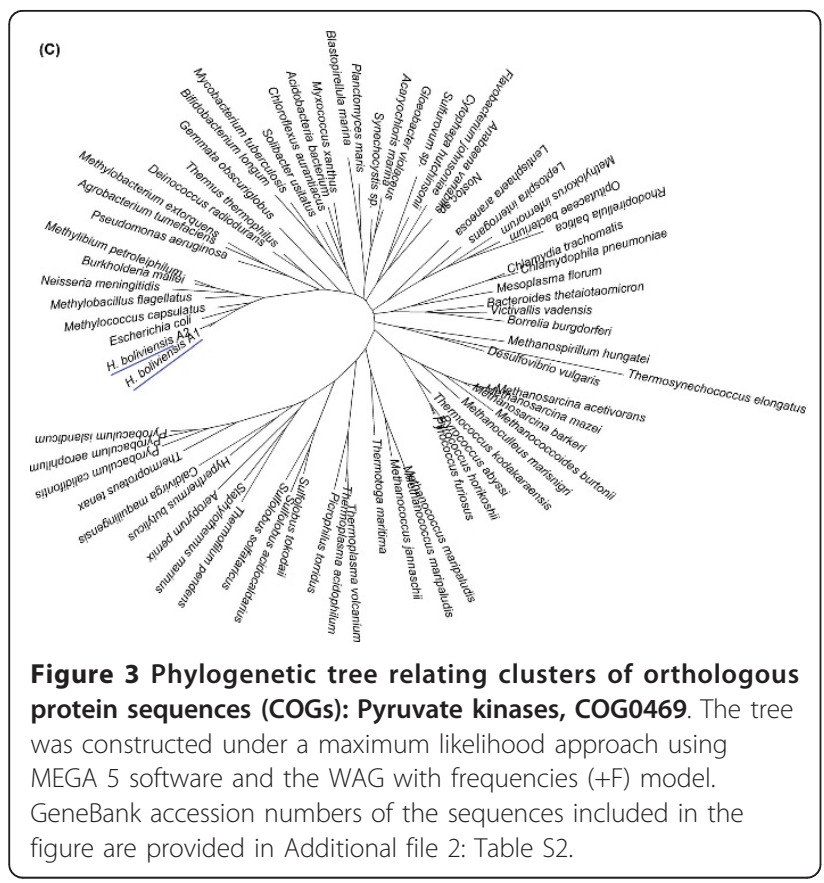

obtained from other bacteria; only a $34 \%$ of the proteins evolved among Proteobacteria (Figure 4). Moreover, transfer of genes from thermophilic organisms and halophilic archaea has had an effect on the genome evolution of $H$. boliviensis as well (Figure 4). Yet, 9\% of the proteins of $H$. boliviensis did not cluster with any of the proteins belonging to the reference microorganisms (Figure 4). They may form phylogenetic groups with proteins of microorganisms that were not included in these studies.

Under the neutral mutation-random drift theory, it is assumed that a certain fraction of new mutation are free of constraint or are selectively neutral, while the rest have deleterious effects and are selectively eliminated [35]. Nevertheless, Figures 1 and 4 imply that most mutations found in proteins related to carbohydrate transport and metabolism were a result of HGT, which agree on some criteria that point out that genetic drift is not sufficient for claiming neutrality [36], and on a resent observation that estimated that about $60 \%$ of the genome evolution of prokaryotes is dominated by HGT [2]. Furthermore, HGT can be related to adaptation of $H$. boliviensis to its environment (Figures 1,2) and might, therefore, be selected to attain an optimum physiological response of the species to its habitat [3]. Yet, nearly neutral mutations could be inferred from Figure 3 and Additional file 1: Table S1, suggesting a continuous evolution of the proteins [1].

\section{Metabolic assimilation of carbohydrates by $\mathrm{H}$. boliviensis}

The metabolic routes in $H$. boliviensis for the assimilation of carbohydrates were obtained by matching the

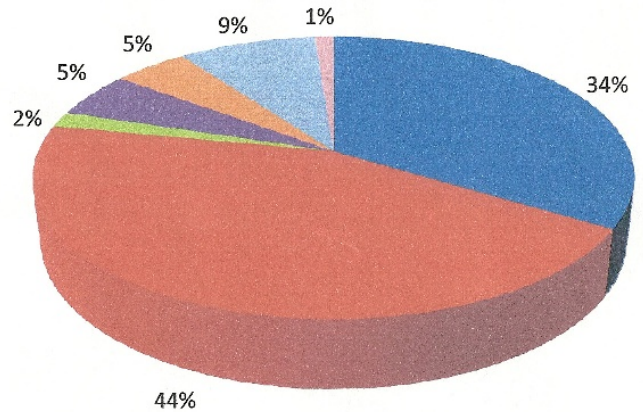

Proteobacteria

Thermophiles

Bacteria-Haloarchaea

Haloarchaea

$$
\begin{aligned}
& \text { Bacteria } \\
& \text { Bacteria-Thermophiles } \\
& \text { No cluster }
\end{aligned}
$$

Figure 4 Percentage of proteins that $H$. boliviensis obtained from other bacteria, halophilic archaea and thermophilic archaea by horizontal gene transfer (HGT), and proteins that evolved among proteins of other Proteobacteria. Some proteins of $\mathrm{H}$. boliviensis did not form a cluster with any of the proteins of the microorganisms used as reference. The proteins analyzed were related to COGs of carbon transport and metabolism as listed in Additional file 1: Table S1.

highest identities of enzymes derived from its genome with the KEGG pathway database [37]. The studies began searching for enzymes of $H$. boliviensis that form part of the starch and sucrose metabolism pathway. Although $H$. boliviensis is unable to hydrolyze starch [15], it assimilates maltose, glucose and other oligosaccharides obtained after the hydrolysis of starch [38]. Furthermore, Halomonas boliviensis can grow on sucrose [15]. We deduced in this study that both maltose and sucrose are metabolized via $\alpha$-D-glucosemono-phosphorylated before entering the glycolysis and gluconeogenesis pathway (Figure 5). Figure 5 shows further a high level of polymorphism for most of the enzymes in this metabolic route.

Similar polymorphism was observed in glycolysis and gluconeogenesis pathways of $H$. boliviensis (Figures 6, 7). For the first part of the metabolism, $H$. boliviensis obtained most of its gens by HGT among bacteria (Figure 6), whereas the enzymes at the bottom of the pathway were mainly related to enzymes of the Proteobacteria (Figure 7). Glycolysis in H. boliviensis concluded with the 2-oxoglutarate dehydrogenase complex (PDHC) (Figure 7 ), that is part of the pyruvate dehydrogenases family. This route is characterized by generation of NADH, and is commonly found in Gram-negative bacteria [39]. The enzymes that form part of PDHC in H. boliviensis were obtained in part from thermophilic archaea and mesophilic bacteria (Figure 7). Archaea do not utilize a pyruvate 


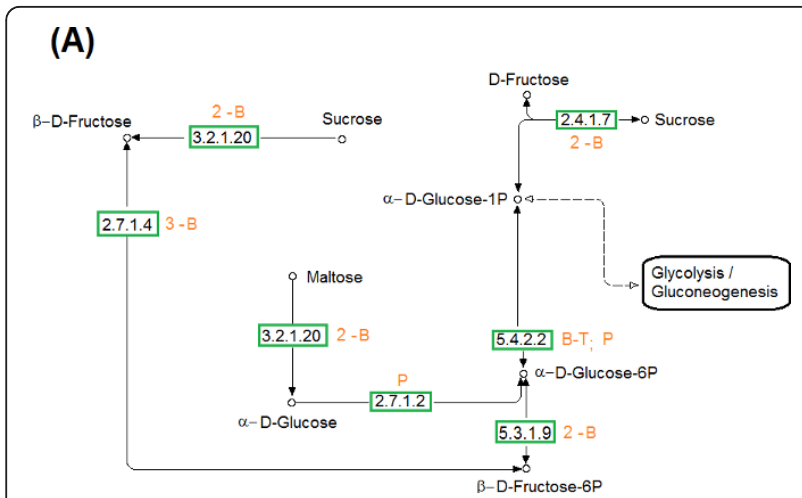

Figure 5 Assimilation of carbohydrates in $\mathrm{H}$. boliviensis by (A) The starch and sucrose metabolism. Numbers and abbreviations for each metabolic step refer to the number of alleles and the cluster that the alleles formed with: P, Proteobacteria; B, bacteria; T, thermophilic archaea; HA, halophilic archaea; A, archaea (non halophilic and non thermophilic) and combinations of these groups of organisms; NC denotes that no cluster was formed between proteins of $\mathrm{H}$. boliviensis and proteins belonging to the microorganisms used as reference. EC numbers for the enzymes in the metabolisms are pointed out as classified in the KEGG pathway database, and are listed in Additional file 3: Table S3 [37].

dehydrogenase complex to transform pyruvate to acetylCoA rather they accomplish the transformation using 2oxoacid oxidoreductases [40]. However, putative enzyme sequences that form part of the PDHC can be found in the genome sequences of thermophilic $[24,25]$ and halophilic archaea $[24,25,41]$. The protein divergence and gene duplication (Figure 3 and 7) may provide evidence of adaptive evolution of the metabolism [13]. The

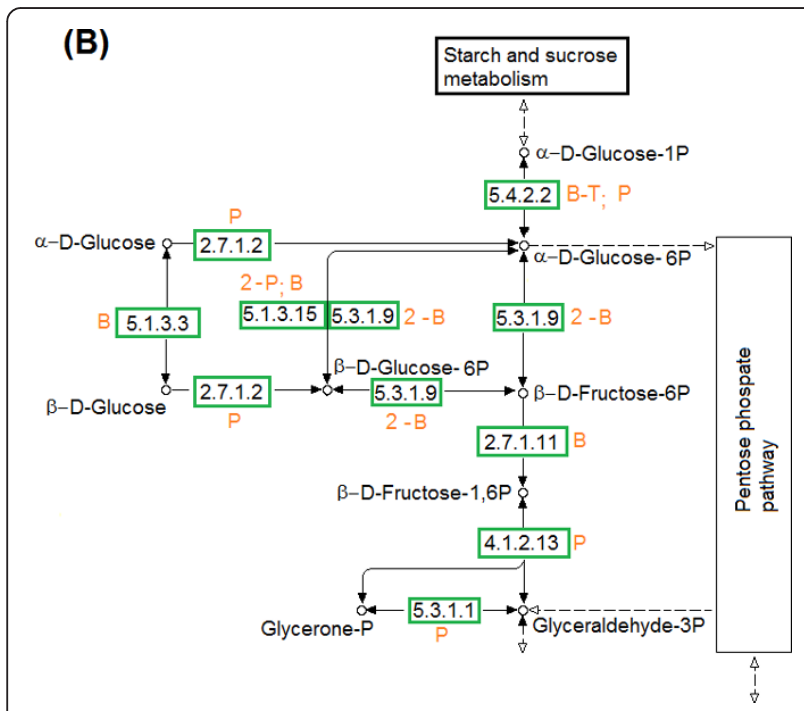

Figure 6 Assimilation of carbohydrates in $H$. boliviensis by (B) The first steps of glycolysis and gluconeogenesis. Name of the enzymes, numbers of alleles and abbreviations are referred as in Figure 5.

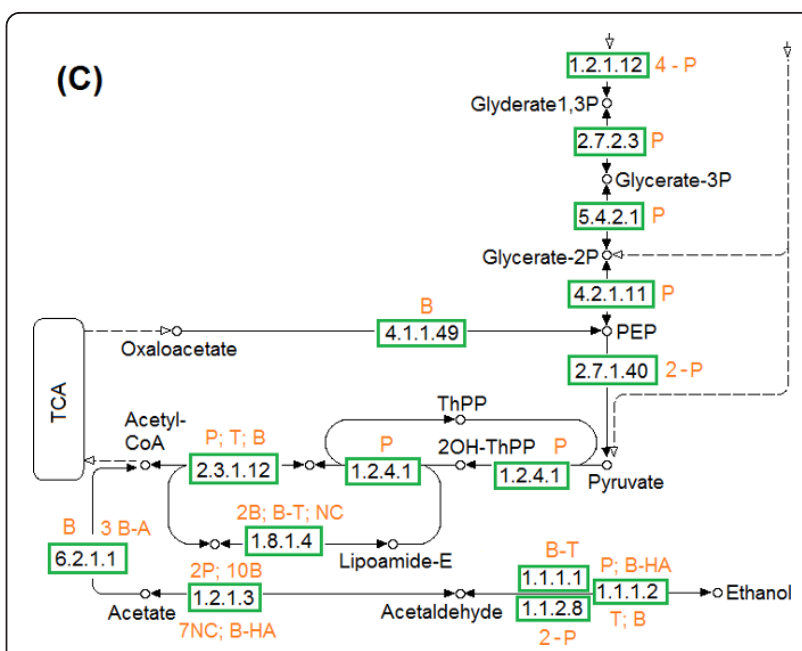

Figure 7 Assimilation of carbohydrates in $\mathrm{H}$. boliviensis by (C) The culmination steps of glycolysis and gluconeogenesis. For the transformation of acetatyl CoA to ethanol, COGs for energy production and conversion and lipid transport and metabolism in cells were included. Name of the enzymes, numbers of alleles and abbreviations are referred as in Figure 5.

diversity of enzymes that can accomplish the same function in $H$. boliviensis explains its versatility in the assimilation of carbohydrates and other carbon sources (e.g. acetate and short chain fatty acids) $[18,38]$. The polymorphic metabolism of $H$. boliviensis might also lead to an efficient generation of energy (ATP) and the reducing agent NADH to correlate with its fast cell growth and its capability to metabolize different carbon sources to PHB $[18,38]$. Besides the synthesis of PHB, excess of NADH could potentially be oxidized by $H$. boliviensis via a fermentative route to allow the formation of ethanol (Figure 7).

\section{Relationship of the enzymes involved in glycolysis and gluconeogenesis among Prokaryotes}

Considering the degree of polymorphism in the metabolic routes of $\mathrm{H}$. boliviensis, we wonder whether this trend could be followed by other microorganisms in its environment. To address this question, supernetworks were constructed by combining the phylogenetic trees related to glycolysis and gluconeogenesis. Figure 8 shows a supernetwork obtained after combining three phylogenetic trees; two of them derived from COGs that are considered among the 102 genes that contain taxonomic information that discriminate well bacteria and archaea in already known families and genera [4]. The internetwork relationship among microorganisms shown in Figure 8 denotes that HGT occurred among bacteria, archaea and between bacteria and archaea. A supernetwork obtained from six trees reflected a higher effect of HGT among microorganisms (Figure 9). In Figure 9, taxonomic differentiation between proteins of bacteria and archaea was barely 


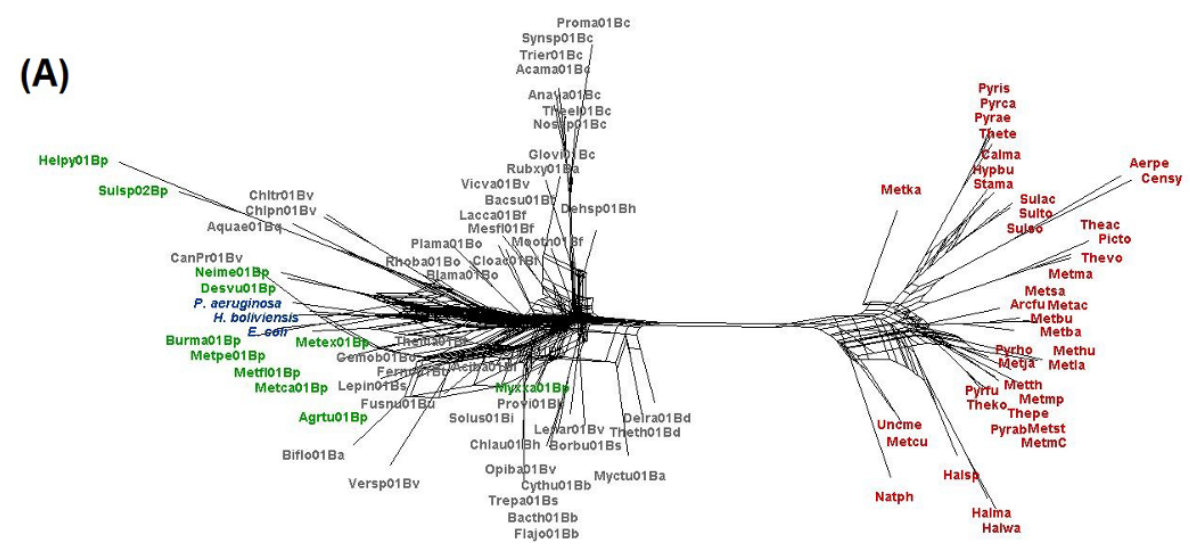

Figure 8 Supernetworks constructed using the Split4tree program after combining phylogenetic trees of proteins of the glycolysis and gluconeogenesis metabolisms in $\mathrm{H}$. boliviensis and reference strains. The figures included combination of trees corresponding to COGs (A) 0126, 0149 and 0837. Names of the archaeal and bacterial species corresponding to each abbreviation used in the figure are listed in Additional file 4: Table S4.

observed, although $H$. boliviensis was still clustered with other $\gamma$-Proteobacteria, i.e. Escherichia coli and Pseudomonas aeruginosa. Finally, 22 phylogenetic trees related to glycolysis and gluconeogenesis were used to attain a supernetwork (Figure 10). Taxonomic differentiation among the proteins of the microorganisms was no longer observed (Figure 10), hence suggesting that flow of genes involved in glycolysis and gluconeogenesis among Prokaryotes was significant. Experimental analysis demonstrated polymorphism for the enzymes that form part of glycolysis in E. coli [42], whereas various different Halomonas and Chromohalobacter species grow on several common carbon sources and are able to produce PHB [43]. On the whole, our studies imply that the availability of the selection of a particular carbohydrate by a microbial species should be related to the rate of evolution of the enzymes, and might be linked not only to the size of the microbial population, as stated by the nearly neutral theory of evolution [1], but also to the groups of microorganisms able to thrive in a particular niche.

\section{Use of combination of carbohydrates for the production of PHB by H. boliviensis}

The aforementioned results revealed that the rate of evolution, mutations and the molecular interaction between $H$. boliviensis and other microorganisms in its environment influenced significantly the evolution of the carbohydrate transport and metabolism in this bacterium-a similar evolutionary pattern might be expected in other prokaryotes. However, phenotypic traits concerning microbial growth on different carbon sources are stamps of different phylogenetic groups and species. We hypothesized that the amount and type of carbon sources in a particular environment should also influence the

(B)

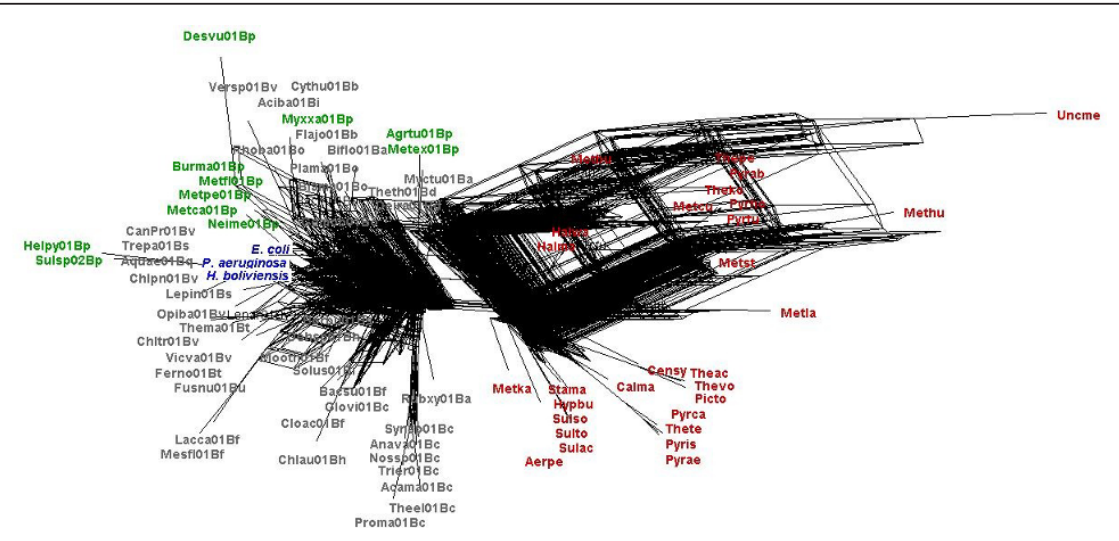

Figure 9 Supernetworks constructed using the Split4tree program after combining phylogenetic trees of proteins of the glycolysis and gluconeogenesis metabolisms in $\mathrm{H}$. boliviensis and reference strains. The figures included combination of trees corresponding to COGs (B) 0126, 0149, 0837, 0469, 0696 and 0837. Names of the archaeal and bacterial species corresponding to each abbreviation used in the figure are listed in Additional file 4: Table S4. 

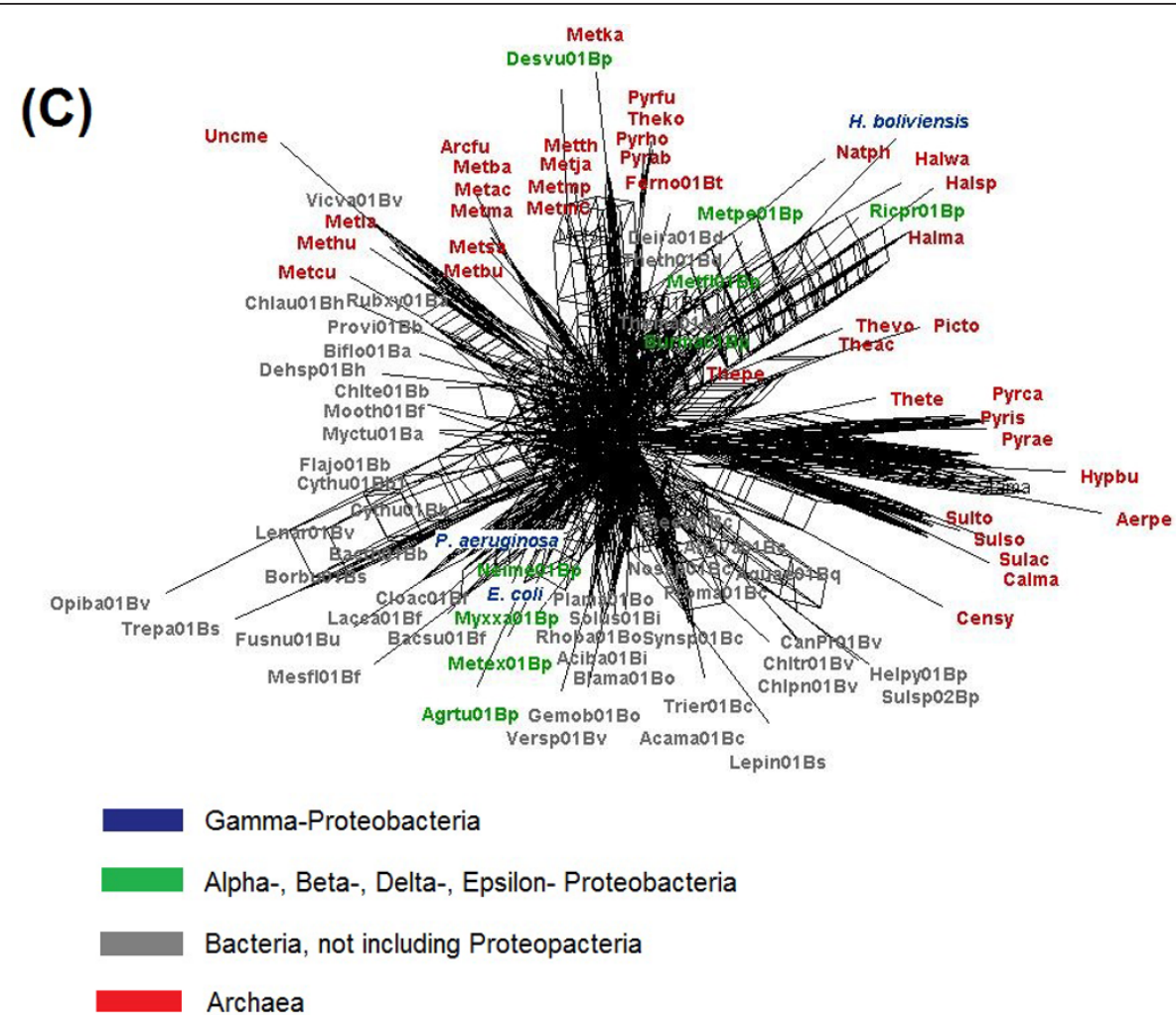

Figure 10 Supernetworks constructed using the Split4tree program after combining phylogenetic trees of proteins of the glycolysis and gluconeogenesis metabolisms in $\mathrm{H}$. boliviensis and reference strains. The figures included combination of trees corresponding to COGs (C) 0057, 0126, 0148, 0149, 0166, 0191, 0205, 0235, 0365, 0469, 0508, 0696, 0837, 1012, 1063, 1109, 1249, 1454, 1866, 2017,2609 and 4993. Names of the archaeal and bacterial species corresponding to each abbreviation used in the figure are listed in Additional file 4: Table S4.

fitness of glycolysis and gluconeogenesis fluxes in $\mathrm{H}$. boliviensis. The influence of the environment on the functional features of enzymes is a context not commonly evaluated in evolutionary theories of metabolic pathways $[12,14]$. For this reason, we decide to use various combinations of glucose and sucrose concentrations as precursors for PHB synthesis in H. boliviensis (Figures 11, 12).

Three alleles of the PHB synthases were found in the genome of $H$. boliviensis (Additional file 5: Figure S1). The three alleles are closely related to PHB synthases of Proteobacteria. Moreover, H. boliviensis A2 was clustered with two alleles of PHB polymerases of Halomonas sp. TD01 (Figure S1); one of these alleles (phaC1) was previously reported [6]. However, a third allele of Halomonas sp. TD01 (named phaC2) showed a distant phylogenetic relationship to the PHB synthases of Proteobacteria (Figure S1); phaC2 might have been acquired by HGT [6]. Research on the PHB polymerization and depolymerization pathways in $H$. boliviensis is in progress. PHB production by $H$. boliviensis was performed in shake flask experiments under nitrogen limitation conditions (i.e. a low concentration sodium glutamate was added to the culture medium to limit the

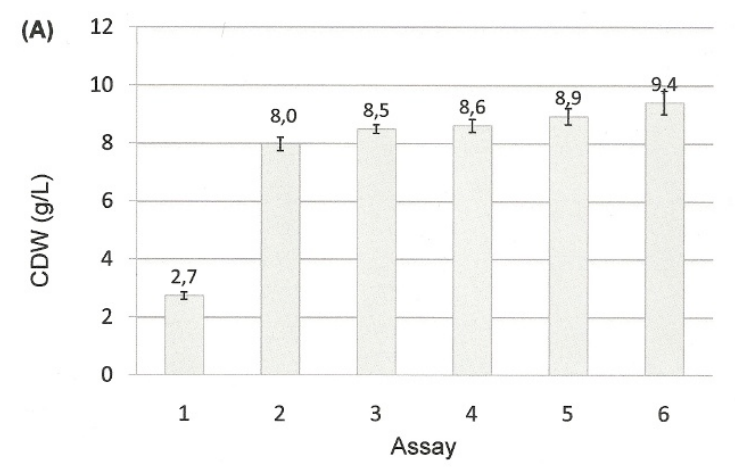

Figure 11 Cell growth and PHB production by $\mathrm{H}$. boliviensis using different combinations of carbohydrates and molasses.

(A) Cell dry weight (CDW) in batch culture using 1.5\% (w/v) sucrose and $1 \%(\mathrm{w} / \mathrm{v})$ glucose. Assay numbers refer to the concentration of carbohydrates added to the medium, \%(w/v): 1) 2.5 sucrose, 2) 2.0 sucrose and 0.5 glucose, 3) 1.5 sucrose and 1 glucose, 4) 1.0 sucrose and 1.5 glucose, 5) 0.3 sucrose, 0.7 glucose and 1.5 dried molasses and 6) 2.5 dried molasses. All experiments were performed in shake flasks at $35^{\circ} \mathrm{C}$ and $220 \mathrm{rpm}$ of agitation. All experiments were performed in triplicate. The error bars refer to the SD of the average values. 


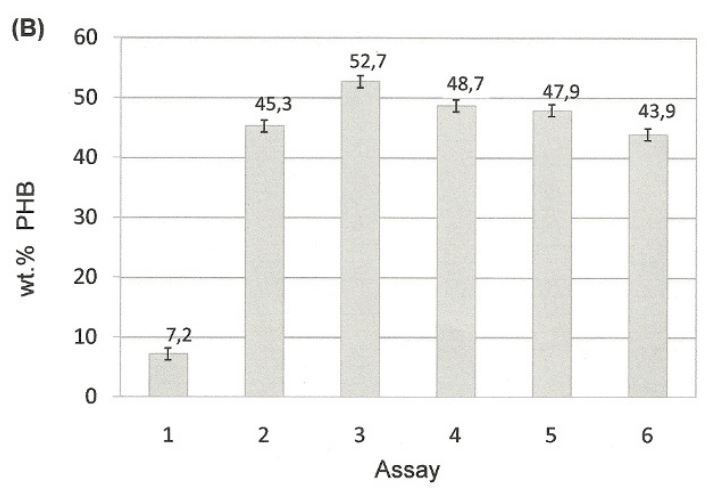

Figure 12 Cell growth and PHB production by $\mathrm{H}$. boliviensis using different combinations of carbohydrates and molasses. (B) PHB accumulation in batch culture using 1.5\% (w/v) sucrose and $1 \%(\mathrm{~W} / \mathrm{V})$ glucose. Assay numbers refer to the concentration of carbohydrates added to the medium, \%(w/v): 1) 2.5 sucrose, 2) 2.0 sucrose and 0.5 glucose, 3) 1.5 sucrose and 1 glucose, 4) 1.0 sucrose and 1.5 glucose, 5) 0.3 sucrose, 0.7 glucose and 1.5 dried molasses and 6) 2.5 dried molasses. All experiments were performed in shake flasks at $35^{\circ} \mathrm{C}$ and $220 \mathrm{rpm}$ of agitation. All experiments were performed in triplicate. The error bars refer to the SD of the average values.

cell growth). When sucrose was used as the sole carbon source, the accumulation of PHB in $H$. boliviensis $(7.2$ wt\%) and cell growth $(2.7 \mathrm{~g} / \mathrm{L})$ were low compared to those obtained with combinations of sucrose and glucose (Figures 11,12). Cell growth increased as the amount of glucose was higher in the medium to reach $8.6 \mathrm{~g} / \mathrm{L}$ (Figure 11), while the maximum PHB content in $H$. boliviensis was $52.7 \mathrm{wt} \%$ when $1.5 \%(\mathrm{w} / \mathrm{v})$ sucrose and $1.0 \%(\mathrm{w} / \mathrm{v})$ glucose were included in the medium composition (Figure 12). The use of molasses enhanced to some extent the cell growth, c.a. $9.4 \mathrm{~g} / \mathrm{L}$, but the PHB accumulated in the cells was lower, $43.9 \mathrm{wt} \%$ (Figures 11, 12). Both the cell density and the maximum PHB yield attained by $H$. boliviensis are higher to those reported using glucose as carbon source, i.e. $5.3 \mathrm{~g} / \mathrm{L}$ and $45 \mathrm{wt} \%$ respectively, under similar culture conditions [16].

Glucose and sucrose uptake and assimilation were analyzed further using the optimum sucrose and glucose ratio, i.e. 1.5:1, for PHB production (Figure 13). Residual cell mass (RCM), which is the cell biomass without the polymer inclusions, was used to analyze the active cell growth. Glucose and sucrose were assimilated parallelly during the exponential phase of growth of $H$. boliviensis (Figure 13). However, glucose consumption rate by $H$. boliviensis shows a linear decrease after 9 hours of cultivation when sodium glutamate was almost depleted from the medium and PHB synthesis was triggered (Figure 13). Sucrose concentration in the medium was constant from

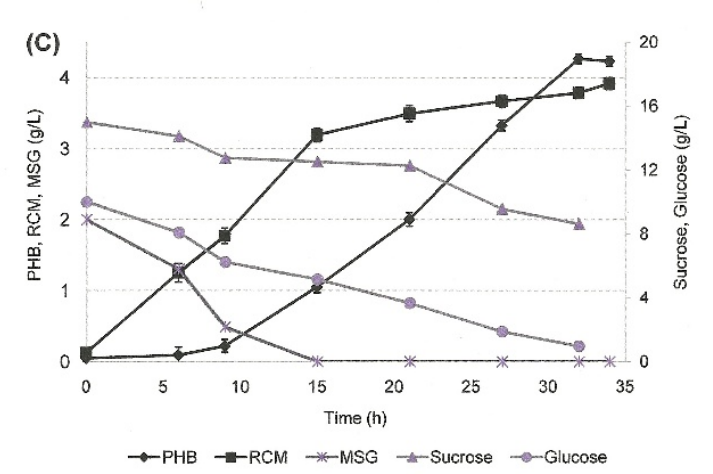

Figure 13 Cell growth and $\mathrm{PHB}$ production by $H$. boliviensis using different combinations of carbohydrates and molasses. (C) Carbohydrate assimilation and PHB production in batch culture using 1.5\% (w/v) sucrose and $1 \%(\mathrm{w} / \mathrm{v})$ glucose. All experiments were performed in shake flasks at $35^{\circ} \mathrm{C}$ and 220 rpm of agitation. The abbreviations RCM and MSG in the figure refer to residual cell mass and mono sodium glutamate, respectively. All experiments were performed in triplicate. The error bars refer to the SD of the average values.

9 to $21 \mathrm{~h}$ of cultivation and was only reduced when the concentration of glucose in the medium was low (Figure 13). Glucose generates a higher amount of energy and NADH in $H$. boliviensis than sucrose because the CDW and PHB reached by $H$. boliviensis using glucose were much higher than those achieved when sucrose was used as the sole carbon source (Figures 11, 12) [16]. Nevertheless, an overflow of NADH is known to obstruct the tricarboxylic acid cycle because of inhibition of citrate synthase [44]. An adequate ratio of glucose and sucrose in the medium promoted an appropriate balance of energy for an active cell growth (Figures 11, 13), albeit Figure 13 reflects that glucose is preferred during the PHB accumulation in $H$. boliviensis due to an excess of NADH in the cytoplasm of cells improves the polymer synthesis $[19,44]$.

The maximum PHB concentration and volumetric productivity reached by $H$. boliviensis were $4.3 \mathrm{~g} / \mathrm{L}$ and 0.13 $\mathrm{g} / \mathrm{L} / \mathrm{h}$, respectively; they are comparable to those reached by Cupriavidus necator, i.e. $5.1 \mathrm{~g} / \mathrm{L}$ and $0.11 \mathrm{~g} / \mathrm{L} / \mathrm{h}$ [45], and to those reported for a recombinant $E$. coli strain, c. a. $7.2 \mathrm{~g} / \mathrm{L}$ and $0.15 \mathrm{~g} / \mathrm{L} / \mathrm{h}$ [46]. The medium for C. necator and $E$. coli contained glucose as the carbon source for experiments performed in shake flasks. Under similar culture conditions, Azotobacter vinelandii led to a PHB concentration of $7.5 \mathrm{~g} / \mathrm{L}$ and a productivity of $0.30 \mathrm{~g} / \mathrm{L} / \mathrm{h}$ [47]. These bacteria attained among the highest productions of $\mathrm{PHB}$, and are recognized for their potential utilization at industrial scales $[19,21]$.

The viability of the commercialization of $\mathrm{PHB}$ is dependent upon the reduction of the total production costs [22]. The price of the carbon source supplied in 
the culture medium may account up to $40 \%$ of the total production costs [22]. Sucrose is at least two times cheaper than glucose while molasses are cheaper than sucrose. The results obtained for the production of PHB by $H$. boliviensis (Figures 11, 13) suggest that an agricultural surplus such as molasses could be used during the bioprocess scale up to stimulate the cell growth; furthermore an optimum ratio of sucrose and glucose should be added in the culture medium of the largest bioreactor used in a process to induce a high polymer production. Replacing partially glucose by sucrose and molasses should surly reduce the production costs of the polymer and lead also to an environmentally friendly bioprocess. Nevertheless, fed-batch cultivations systems are yet to be performed with $H$. boliviensis using combinations of carbohydrates to reveal their potential in a large scale process.

\section{Conclusions}

The genome size and number of genes found in $\mathrm{H}$. boliviensis were similar to those determined for other halophilic bacteria of the family Halomonadaceae. The ability of $H$. boliviensis to grow on different carbon sources is explained by the high number of genes related to the carbohydrate uptake and metabolism. Interestingly, most of these genes were obtained from other bacteria by HGT, only $34 \%$ of the genes evolved as proteins belonging to Proteobacteria, while $13 \%$ of the genes were transferred from haloarchaea and thermophilic archaea. Furthermore, the diversity of enzymes that have the same physiological function led to polymorphism in the metabolic routs. Results obtained in this article depict that most genetic modifications and enzyme polymorphism in the genome of $H$. boliviensis were mainly influenced by HGT rather than nearly neutral mutations. Molecular adaptation and evolution experienced by $H$. boliviensis were also a response to environmental conditions such as the type and amount of carbohydrates in its ecological niche. Consequently, the genome evolution of $H$. boliviensis showed to be strongly influenced by the type of microorganisms, genetic interaction among microbial species and its environment. Such trend should also be experienced by other prokaryotes. A system for PHB production by $H$. boliviensis that takes into account the evolutionary adaptation of this bacterium to the assimilation of combinations of carbohydrates suggests the feasibility of a bioprocess economically viable and environmentally friendly.

\section{Halomonas boliviensis genome sequence}

This whole genome shotgun project was deposited at DDBJ, EMBL and GenBank under the accession number AGQZ00000000. The version described in this paper is the first version, AGQZ01000000.

\section{Additional material}

Additional file 1: Table S1. Clusters of orthologous genes (COGs) of $\mathrm{H}$. boliviensis related to carbon transport and metabolism.

Additional file 2: Table S2. List of GenBank accession numbers for the microorganisms shown in the phylogenetic trees in Figure 1, 2, 3.

Additional file 3: Table S3. EC numbers and COG classification of the enzymes involved in starch metabolism, glycolysis and gluconeogenesis in $\mathrm{H}$. boliviensis.

Additional file 4: Table S4. List of species and abbreviations of the 100 microorganisms (59 Bacteria and 41 Archaea) used as reference. Abbreviations are named as described by Puigbò, et al. 2009.

Additional file 5: Figure S1.

\section{Acknowledgements}

The authors would like to thank the Swedish International Development Cooperation Agency (Sida) for supporting our research work.

\section{Author details}

${ }^{1}$ Centro de Biotecnología, Facultad de Ciencias y Tecnología, Universidad Mayor de San Simón, Cochabamba, Bolivia. ²Department of Biotechnology, Lund University, P.O. Box 124, SE-221 00 Lund, Sweden.

\section{Authors' contributions}

$\mathrm{DG}, \mathrm{AB}-\mathrm{S}, \mathrm{CC}-\mathrm{O}, \mathrm{MG}-\mathrm{M}$ and $\mathrm{JQ}$ performed the evolutionary analyses on the COGs corresponding to the carbohydrate transport and metabolism of $\mathrm{H}$. boliviensis. JQ constructed the supernetworks. JQ, DG and NC-Q studied the $\mathrm{PHB}$ production by $\mathrm{H}$. boliviensis. All authors wrote the manuscript and approved the final version.

\section{Competing interests}

The authors declare that they have no competing interests.

Received: 2 January 2012 Accepted: 17 April 2012

Published: 17 April 2012

\section{References}

1. Ohta T: The nearly neutral theory of molecular evolution. Annu Rev Ecol Syst 1992, 23:263-286.

2. Puigbò $\mathrm{P}$, Wolf $\mathrm{YI}, \mathrm{Koonin} \mathrm{EV}$ : The tree and net components of prokaryote evolution. Genome Biol Evol 2010, 2:745-756.

3. Orr H: The genetic theory of adaptation: a brief history. Nature Reviews 2005, 6:119-127.

4. Puigbò $P$, Wolf YI, Koonin EV: Search for a 'Tree of Life' in the thicket of the phylogenetic forest. J Biology 2009, 8:1-17.

5. Schliep K, Lopez P, Lapointe FJ, Bapteste E: Harvesting evolutionary signals in a forest of prokaryotic gene trees. Mol Biol Evol 2010, 28:1393-1405.

6. Cai L, Tan D, Aibaidula G, Dong X, Chen J, Tian W, Chen G: Comparative genomics study of polyhydroxyalkanoates $(\mathrm{PHA})$ and ectoine relevant genes from Halomonas sp. TD01 revealed extensive horizontal gene transfer events and co-evolutionary relationships. Microb Cell Fact 2011, 10:88.

7. Oren A: Microbial life at high salt concentrations: phylogenetic and metabolic diversity. Saline Systems 2008, 4:2.

8. Oren A, Larimer F, Richardson P, Lapidus A, Csonka LN: How to be moderately halophilic with broad salt tolerance: clues from the genome of Chromohalobacter salexigens. Extremophiles 2005, 9:275-279.

9. Schvibbert $K$, et al: A blueprint of ectoine metabolism from the genome of the industrial producer Halomonas elongata DSM $2581^{\top}$. Environ Microbiol 2010, 13:1973-1994.

10. Lin $Y$, et al: Draft genome sequence of Halomonas sp. strain HAL1, a moderately halophilic arsenite-oxidizing bacterium isolated from goldmine soil. J Bacteriol 2011, 194:199-200.

11. Roberts MF: Organic compatible solutes of halotolerant and halophilic microorganism. Saline Systems 2005, 1:5.

12. Eanes WF: Molecular population genetics and selection in the glycolytic pathway. J Exp Biol 2011, 214:165-171. 
13. Eanes WF: Analysis of selection on enzyme polymorphism. Annu Rev Ecol Syst 1999, 30:301-326.

14. Hartl D, Dykhuizen D, Dean A: Limits of adaptation: the evolution of selective neutrality. Genetics 1985, 111:655-674.

15. Quillaguamán J, Hatti-Kaul R, Mattiasson B, Alvarez MT, Delgado O: Halomonas boliviensis sp. nov., an alkalitolerant, moderate halophile bacterium isolated from soil around a Bolivian hypersaline lake. Int J Syst Evol Microbiol 2004, 54:721-725.

16. Quillaguamán J, Van-Thuoc D, Guzmán H, Guzmán D, Martín J, Akaraonye E, Hatti-Kaul R: Poly(3-hydroxybutyrate) production by Halomonas boliviensis in fed-batch culture. App/ Microbiol Biotechnol 2008, 78:227-232.

17. Van-Thuoc D, Guzmán H, Quillaguamán J, Hatti-Kaul R: High productivity of ectoines by Halomonas boliviensis using a combined two-step fed-batch culture and milking process. J Biotechnol 2010, 147:46-51.

18. Quillaguamán J, Delgado O, Mattiasson B, Hatti-Kaul R: Poly( $\beta$ hydroxybutyrate) production by a moderate halophile, Halomonas boliviensis LC1. Enzyme Microb Technol 2006, 38:148-154.

19. Steinbüchel A, Füchtenbush B: Bacterial and other biological systems for polyester production. Trends Biotechnol 1998, 16:419-427.

20. Philip S, Keshavarz T, Roy I: Polyhydroxyalkanoates: biodegradable polymers with a range of applications. J Chem Technol Biotechnol 2007, 82:233-247.

21. Lee SY: Plastic bacteria? Progress and prospects for polyhydroxyalkanoate production in bacteria. Trends Biotechnol 1996, 14:431-438.

22. Choi J, Lee S: Factors affecting the economics of polyhydroxyalkanoate production by bacterial fermentation. Appl Microbiol Biotechnol 1998, 51:13-21.

23. Delcher A, Bratke $K$, Powers E, Salzberg S: Identifying bacterial genes and endosymbiont DNA with Glimmer. Bioinformatics 2007, 23:673-679.

24. Tatusov $R$, et al: The COG database: an updated version includes eukaryotes. BMC Bioinforma 2003, 4:41.

25. Jensen $L$, Julien $P$, Kuhn M, von Mering C, Muller J, Doerks T, Bork P: eggNOG: automated construction and annotation of orthologous groups of genes. Nucleic Acids Res 2008, 36:D250-D254

26. Edgar R: MUSCLE: multiple sequence alignment with high accuracy and high throughput. Nucleic Acids Res 2004, 32:1792-1797.

27. Tamura K, Peterson D, Peterson N, Stecher G, Nei M, Kumar S: MEGA5: molecular evolutionary genetics analysis using maximum likelihood, evolutionary distance, and maximum parsimony methods. Mol Biol Evol 2011, 10:2731-2739.

28. Huson D, Bryant D: Application of phylogenetic networks in evolutionary studies. Mol Biol Evol 2006, 23:254-267.

29. Huson D, Dezulian T, Klopper T, Steel M: Phylogenetic supernetworks from partial trees. IEEE/ACM Trans Comput Biol Bioinform 2004, 1:151-158.

30. Lee SY, Wong HH, Choi J, Lee SH, Lee SC, Han CS: Production of mediumchain-length polyhydroxyalkanoates by high-cell-density cultivation of Pseudomonas putida under phosphorus limitation. Biotechnol Bioeng 2000, 68:466-470.

31. Onraedt A, Wlcarius B, Soetaert W, Vandamme E: Optimization of ectoine synthesis through fed-batch fermentation of Brevibacterium epidermis. Biotechnol Prog 2005, 21:1206-1212

32. Arahal DR, García MT, Vargas C, Cánovas D, Nieto JJ, Ventosa A: Chromohalobacter salexigens sp. nov., a moderately halophilic species that includes Halomonas elongata DSM 3043 and ATCC 33174. Int J Syst Evol Microbiol 2001, 51:1457-1462

33. Arahal DR, Ventosa A: The family Halomonadaceae. In The Prokaryotes. A handbook on the biology of bacteria. Edited by: Dworkin M, et al. New York: Springer; 2006:811-835

34. Gandbhir M, Rashed I, Marlière P, Mutzel R: Convergent evolution of amino acid usage in archaebacterial and eubacterial linages adapted to high salt. Res Microbiol 1995, 146:113-120.

35. Kimura M: Evolutionary rate at the molecular level. Nature 1968, 217:624-626.

36. Kreitman $\mathrm{M}$ : The neutral theory is dead. Long live the neutral theory. Bioessays 1996, 18:678-683.

37. Kanehisa $\mathrm{M}$, et al: From genomics to chemical genomics: new developments in KEGG. Nucleic Acids Res 2006, 34:D354-D357.

38. Quillaguamán J, Hashim S, Bento F, Mattiasson B, Hatti-Kaul R: Poly( $\beta$ hydroxybutyrate) production by a moderate halophile, Halomonas boliviensis LC1 using starch hydrolysate as substrate. J App/ Microbiol 2005, 99:151-157.

39. de Kok A, Hengeveld AF, Martin A, Westphal AH: The pyruvate dehydrogenase multi-enzyme comples from Gram-negative bacteria. Biochim Biophys Acta 1998, 1385:353-366.

40. Danson MJ: Central metabolism of the Archaea. New Compr Biochem 1993, 26:1-24

41. Jolley KA, et al: 2-oxoacid dehydrogenase mutienzyme complexes in the halophilic Archaea? Gene sequences and protein structural predictions. Microbiology 2000, 146:1061-1069.

42. Sawyer S, Dykhuizen D, Hartl D: Confidence interval for the number of selectively neutral amino acid polymorphism. Proc Natl Acad Sci USA 1987, 84:6225-6228.

43. Quillaguamán J, Guzmán H, Van-Thuoc D, Hatti-Kaul R: Synthesis and production of polyhydroxyalkanoates by halophiles: current potential and future prospects. App/ Microbiol Biotechnol 2010, 85:1687-1696.

44. Babel W, Ackermann JU, Breuer U: Physiology, regulation, and limits of the synthesis of poly (3HB). In Advances in Biochemical Engineering/ Biotechnology: Biopolyesters. Edited by: Scheper T, Babel W, Steinbüchel A. Berlin: Springer; 2001:125-157.

45. Doi Y, Tamaki A, Kunioka M, Soga K: Production of copolyesters of 3hydroxybutyrate and 3-hydroxyvalerate by Alcaligenes eutrophus from butyric and pentanoic acids. Appl Microbiol Biotechnol 1988, 28:330-334

46. Lee SY, Lee KM, Chang HN, Steinbüchel A: Comparison of recombinant Escherichia coli strains for synthesis and accumulation of poly-(3hydroxybutyric acid) and morphological changes. Biotechnol Bioeng 1994, 44:1337-1347.

47. Page WJ: Production of poly- $\beta$-hydroxybutyrate by Azotobacter vinelandii UWD in media containing sugars and complex nitrogen. Appl Microbiol Biotechnol 1992, 38:117-121.

doi:10.1186/2046-9063-8-9

Cite this article as: Guzmán et al:: Evolutionary patterns of carbohydrate transport and metabolism in Halomonas boliviensis as derived from its genome sequence: influences on polyester production. Aquatic Biosystems 2012 8:9.

\section{Submit your next manuscript to BioMed Central and take full advantage of:}

- Convenient online submission

- Thorough peer review

- No space constraints or color figure charges

- Immediate publication on acceptance

- Inclusion in PubMed, CAS, Scopus and Google Scholar

- Research which is freely available for redistribution

Submit your manuscript at www.biomedcentral.com/submit
C) Biomed Central 